\title{
THE d'-DIBARYON IN THE NONRELATIVISTIC QUARK MODEL
}

\author{
A. J. BUCHMANN, GEORG WAGNER, K. TSUSHIMA, AMAND FAESSLER \\ Institute for Theoretical Physics, University of Tübingen \\ D-72076 Tübingen, Germany \\ and \\ L. YA. GLOZMAN \\ Institute for Theoretical Physics, University of Graz \\ A-8010 Graz, Austria
}

\begin{abstract}
The narrow peak recently found in various pionic double charge exchange (DCX) cross sections can be explained by the assumption of a universal resonance at $2065 \mathrm{MeV}$, called $d^{\prime}$. We calculate the mass of a six-quark system with $J^{P}=0^{-}, T=0$ quantum numbers employing a cluster model and a shell model basis to diagonalize the nonrelativistic quark model Hamiltonian.
\end{abstract}

\section{Introduction}

Recently, a very narrow peak $\left(\Gamma_{\text {medium }} \approx 5 \mathrm{MeV}\right)$ has been observed in the $\left(\pi^{+}, \pi^{-}\right)$double charge exchange cross section at an incident pion energy of $T_{\pi}=50 \mathrm{MeV}$ and forward angles $\Theta=5^{\circ}$. 1 . Dedicated DCX experiments on a number of nuclei ranging from ${ }^{12} \mathrm{C}$ to ${ }^{56} \mathrm{Fe}$ have shown that the position and width of this peak is largely independent of the nuclear target. Therefore, a fundamental two-nucleon or six-quark process seems to be involved. In fact, due to charge conservation the DCX reaction involves at least two nucleons within the nucleus and is quite sensitive to short-range $N N$ correlations . Conventional DCX calculations have so far been unable to explain these experimental results 3 . On the other hand, the assumption of a single narrow resonance, called $d^{\prime}$, with spin, parity $J=0^{-}$and isospin $T=0$ and a resonance energy of $M_{d^{\prime}}=2065 \mathrm{MeV}$ works extremely well in describing all available DCX data 1 . The mass of the $d^{\prime}$-resonance coincides with $M_{d^{\prime}}=2 M_{N}+m_{\pi}+T_{\pi}$ 国. The small decay width of the $d^{\prime}$ is naturally explained by its small mass which is below any baryon-baryon $\left(N N^{*}, N^{*} N^{*}, \ldots\right)$ threshold and by its quantum numbers $\mathrm{J}^{P}=0^{-}$and $T=0$ which prevent a decay into the nucleon-nucleon channel.

QCD does not exclude a rearrangement of the six quarks into more exotic configurations such as a diquark $\left(q^{2}\right)$ and a tetraquark $\left(q^{4}\right)$ cluster. In fact, the bag-string model 6 predicts that a diquark cluster with spin (isospin) $S=0(T=0)$ and a tetraquark cluster with spin (isospin) $S=1(T=0)$ moving with relative angular momentum $L=1$ is energetically the most favorable configuration for a system with $d^{\prime}$ quantum numbers. Ref ${ }^{6}$ predicts a $d^{\prime}$ mass of $M_{d^{\prime}} \approx 2100 \mathrm{MeV}$. However, in these calculations the quark exchange interactions between the clusters have been neglected. Therefore, we perform a calculation

\footnotetext{
${ }^{*}$ It has been suggested that the $d^{\prime}$-dibaryon is a $\pi N N$ resonance with isospin $T=2$. However, the $T=2$ assignment seems to be in conflict with experimental information from other sources 5 .
} 
in the nonrelativistic quark model (NRQM) in which the complications due to the Pauli principle are properly taken into account. We use two different sets of basis states (i) a cluster model basis using the Resonating Group Method (RGM), (ii) a quark shell-model basis. Furthermore, unlike the bag-string model, the NRQM calculation includes the nonperturbative $\pi$ - and $\sigma$-meson exchange interactions between quarks as required by the spontaneously broken chiral symmetry of QCD.

\section{The nonrelativistic quark potential model}

In the NRQM a system of $n$-quarks with equal masses $m_{q}=313 \mathrm{MeV}$ is described by the Hamiltonian

$$
H=\sum_{i=1}^{n}\left(m_{q}+\frac{\mathbf{p}_{i}^{2}}{2 m_{q}}\right)-\frac{\mathbf{P}^{2}}{n\left(2 m_{q}\right)}+\sum_{i<j}^{n} V^{\text {conf }}\left(\mathbf{r}_{i}, \mathbf{r}_{j}\right)+\sum_{i<j}^{n} V^{r e s}\left(\mathbf{r}_{i}, \mathbf{r}_{j}\right),
$$

where $\mathbf{r}_{i}, \mathbf{p}_{i}$ are the spatial and momentum coordinates of the i-th quark, respectively and $\mathbf{P}$ is the total momentum of the $n$-quark system. The exact removal of the kinetic energy of the center of mass motion by the third term is crucial.

The long-range confining force is generated by a two-body harmonic oscillator confinement potential

$$
V^{\text {conf }}\left(\mathbf{r}_{i}, \mathbf{r}_{j}\right)=-a_{c} \boldsymbol{\lambda}_{i} \cdot \boldsymbol{\lambda}_{j}\left(\mathbf{r}_{i}-\mathbf{r}_{j}\right)^{2},
$$

where $\boldsymbol{\lambda}_{i}$ is the Gell-Mann matrix of $S U(3)_{\text {color }}$ of the i-th quark. The residual interaction models the relevant properties of QCD, namely asymptotic freedom at short distances and spontaneous chiral symmetry breaking which has important consequences for the short- and intermediate-range interaction between quarks. The one-gluon exchange potential

$$
V^{O G E P}\left(\mathbf{r}_{i}, \mathbf{r}_{j}\right)=\frac{\alpha_{s}}{4} \boldsymbol{\lambda}_{i} \cdot \boldsymbol{\lambda}_{j}\left\{\frac{1}{r}-\frac{\pi}{m_{q}^{2}}\left(1+\frac{2}{3} \boldsymbol{\sigma}_{i} \cdot \boldsymbol{\sigma}_{j}\right) \delta(\mathbf{r})\right\}
$$

provides an effective quark-quark interaction that has the spin-color structure of QCD at short distances. Here, $\mathbf{r}=\mathbf{r}_{i}-\mathbf{r}_{j}$ and $\boldsymbol{\sigma}_{i}$ is the usual Pauli spin matrix.

It is well known that the spontaneous breaking of chiral symmetry by the physical vacuum is responsible for the constituent quark mass generation 8 , as well as for the appearence of almost massless pseudoscalar Goldstone bosons and massive scalar mesons that couple to the constituent quarks. In the NRQM, this is modeled by regularized one-pion and one-sigma exchange potentials between constituent quarks 9,10 .

$$
\begin{aligned}
V^{O P E P}\left(\mathbf{r}_{i}, \mathbf{r}_{j}\right) & =\frac{g_{\pi q}^{2}}{4 \pi} \frac{1}{4 m_{q}^{2}} \frac{\Lambda^{2}}{\Lambda^{2}-m_{\pi}^{2}} \boldsymbol{\tau}_{i} \cdot \boldsymbol{\tau}_{j} \boldsymbol{\sigma}_{i} \cdot \boldsymbol{\nabla}_{r} \boldsymbol{\sigma}_{j} \cdot \nabla_{r}\left(\frac{e^{-m_{\pi} r}}{r}-\frac{e^{-\Lambda r}}{r}\right) \\
V^{O S E P}\left(\mathbf{r}_{i}, \mathbf{r}_{j}\right) & =-\frac{g_{\sigma q}^{2}}{4 \pi} \frac{\Lambda^{2}}{\Lambda^{2}-m_{\sigma}^{2}}\left(\frac{e^{-m_{\sigma} r}}{r}-\frac{e^{-\Lambda r}}{r}\right)
\end{aligned}
$$

with

$$
\frac{g_{\sigma q}^{2}}{4 \pi}=\frac{g_{\pi q}^{2}}{4 \pi}, \quad m_{\sigma}^{2}=\left(2 m_{q}\right)^{2}+m_{\pi}^{2}, \quad \Lambda_{\pi}=\Lambda_{\sigma} \equiv \Lambda
$$

The $\pi q$ coupling constant is simply related to the $\pi N$ coupling constant 9, 10. We use $f_{\pi N}^{2} / 4 \pi=0.074911$. The $\pi q$ cut-off mass $\Lambda$ describes the extended pion-quark vertex. Here, $\Lambda=4.2 \mathrm{fm}^{-1}$ which results in a soft $\pi N$ form factor 12 . 
We take a harmonic oscillator wave function for the i-th quark inside the nucleon and $\Delta$

$$
\varphi^{N(\Delta)}\left(\mathbf{r}_{i}\right)=\left(\frac{1}{\pi b^{2}}\right)^{3 / 4} \exp \left(-\frac{1}{2 b^{2}} \mathbf{r}_{i}^{2}\right)
$$

where $b$ is the harmonic oscillator constant.

Table 1: Quark model parameters. Set I: with regularized $\pi$ - and $\sigma$-meson exchange potentials. The $\sigma$ parameters are fixed by eq.(可). Set II: without $\pi$ - and $\sigma$-exchange potentials 12. Set III: as set I but with reduced confinement strength (see sect. 4).

\begin{tabular}{|r|r|r|r|r|r|r|}
\hline Set & $b[\mathrm{fm}]$ & $\alpha_{s}$ & $a_{c}\left[\mathrm{MeV} \mathrm{fm}^{-2}\right]$ & $m_{\sigma}[\mathrm{MeV}]$ & $g_{\sigma}^{2} /(4 \pi)$ & $\Lambda\left[\mathrm{fm}^{-1}\right]$ \\
\hline I & 0.595 & 0.958 & 13.66 & 626 & 0.554 & 4.2 \\
\hline II & 0.603 & 1.540 & 24.94 & - & - & - \\
\hline III & 0.595 & 0.958 & 5.00 & 626 & 0.554 & 4.2 \\
\hline
\end{tabular}

We determine the parameters $a_{c}, \alpha_{s}$, and $b$ by requiring that the $N(939)$ and $\Delta(1232)$ masses are reproduced and that the nucleon mass is stable with respect to variations in $b$.

$$
M_{N}(b)=3 m_{q}=939 M e V, \quad M_{\Delta}-M_{N}=293 M e V, \quad \frac{\partial M_{N}(b)}{\partial b}=0 .
$$

\section{Six-Quark Models for the d'-Dibaryon}

In the bag-string model, dibaryons are described as rotating strings with colored quark clusters at the ends 6 . This model leads to a linear Regge trajectory of excited states. It predicts that the lowest $L=1$ excited state is obtained for a diquark-tetraquark configuration at around $2100 \mathrm{MeV} 6$.

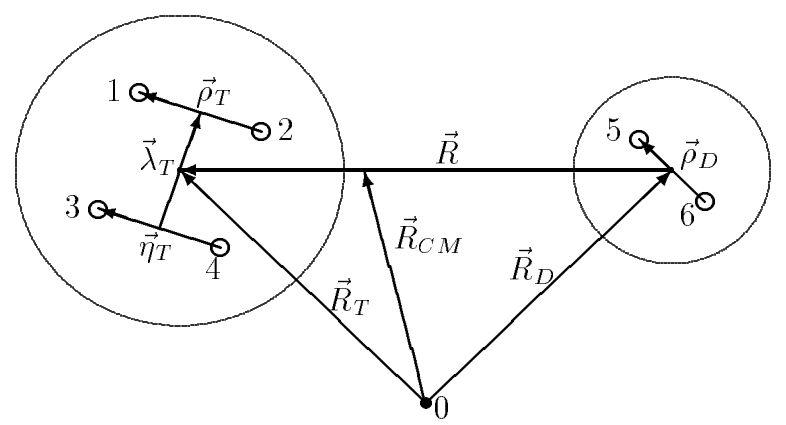

Figure 1: The d'-dibaryon in the $q^{4}-q^{2}$ quark cluster model.

The drawback of this approach is that it does not respect the Pauli principle. Only the quarks within the individual clusters are antisymmetrized but not the quarks belonging to 
different clusters. This is a good approximation for high angular momentum states because in this case the system is fairly elongated and the probability of cluster overlap is small. On the other hand, for a low lying $L=1$ excitation, such as the $d^{\prime}$ one expects a considerable amount of quark exchange between the two clusters. The confining forces between the

Table 2: The mass $\left(M_{d^{\prime}}\right)$ and size $\left(b_{6}\right)$ of the $d^{\prime}$ in the quark cluster model. The masses of the diquark and tetraquark are also given.

\begin{tabular}{|c||c|c|c|c|}
\hline & $M_{2 q}$ & $M_{4 q}$ & $M_{d^{\prime}}$ & $b_{6}$ \\
Set I & 645 & 1455 & 2440 & 0.76 \\
Set II & 637 & 1501 & 2634 & 0.70 \\
Set III & 621 & 1309 & 2111 & 0.95 \\
\hline
\end{tabular}

colored quarks prevent large separations of the clusters and the typical size of such a system is expected to be about $1 \mathrm{fm}$. From our experience with the $N N$ system we know that the Pauli principle plays an important role at such distances 13.

\subsection{The Quark Cluster Model of the d'-Dibaryon}

In this model, the $d^{\prime}$ is described as a nonrelativistic six-quark system in which the quarks interact via the two-body potentials of eq.(価). Tensor and spin-orbit interactions have been omitted since it has previously been shown that they give a negligible contribution to the $d^{\prime}$ mass 14, 15. The six-quark wave function is expanded into the cluster basis

$$
\begin{aligned}
\mid \Psi_{d^{\prime}}^{J=0, T=0}>= & \mathcal{A} \mid\left[\left[\Phi_{T}^{S_{T}=1, T_{T}=0}\left(\boldsymbol{\rho}_{T}, \boldsymbol{\lambda}_{T}, \boldsymbol{\eta}_{T}\right) \times \square\right.\right. \\
& \left.\left.\left.\otimes \Phi_{D}^{S_{D}=0, T_{D}=0}\left(\boldsymbol{\rho}_{D}\right) \times \square_{C}\right]^{S=1, T=0} \otimes \chi_{L=1}(\mathbf{R})\right]^{J=0, T=0} \stackrel{\square}{\square_{C}}\right\rangle,
\end{aligned}
$$

where $\Phi_{T}^{S_{T}=1, T_{T}=0}\left(\boldsymbol{\rho}_{T}, \boldsymbol{\lambda}_{T}, \boldsymbol{\eta}_{T}\right)$ and $\Phi_{B}^{S_{D}=0, T_{D}=0}\left(\boldsymbol{\rho}_{D}\right)$ are the internal wave functions of the tetraquark (T) and diquark (D) clusters, respectively and $\chi_{L=1}(\mathbf{R})$ is the wave function of the relative motion of the two clusters. We use the same harmonic oscillator parameter for the internal and relative motion wave functions. The Young diagrams in eq.(8) show that two color triplet clusters $[211]_{C}$ and $[11]_{C}$ are coupled to a $[222]_{C}$ color-singlet sixquark state. Furthermore, they show that the tetraquark and $d^{\prime}$ wave function are not fully antisymmetric but have mixed symmetry in color space. This nonfactorizability of the color space considerably complicates the calculation.

The advantage of the cluster model is that it provides a continuous transition from the $q^{6}$ six-quark state to the $q^{4}-q^{2}$ clusterized state by smoothly going through all intermediate configurations. There is no rigid and artificial boundary between these extreme configurations; everything is contained in one and the same RGM wave function. This important property is a direct consequence of the Pauli principle on the quark level, which is ensured by the antisymmetrizer $\mathcal{A}$

$$
\mathcal{A}=1-8 P_{46}^{O S T C}+6 P_{35}^{O S T C} P_{46}^{O S T C},
$$




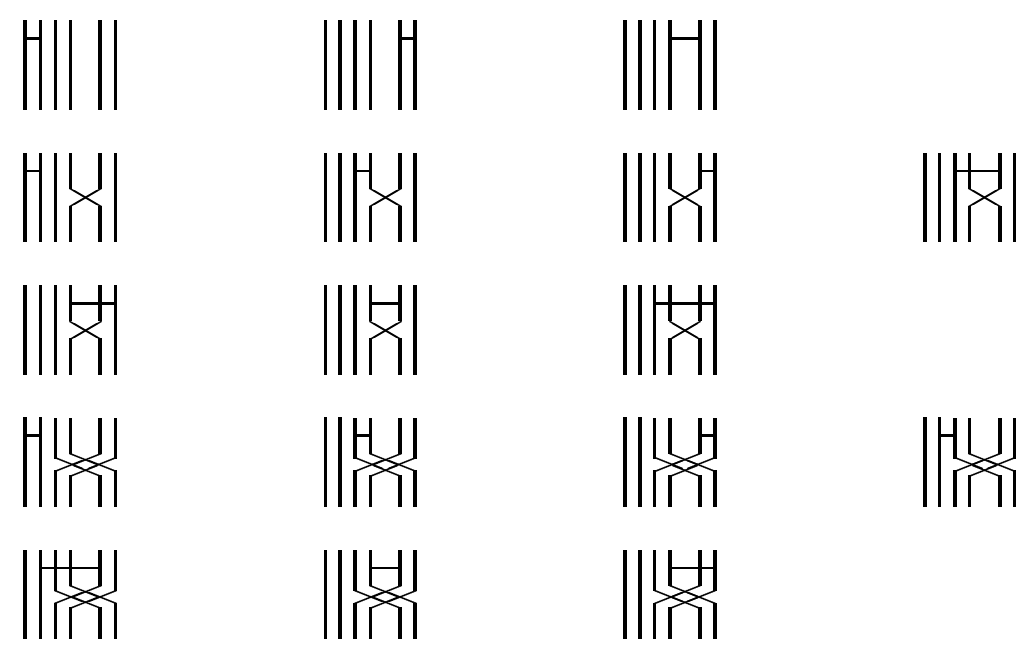

Figure 2: The direct, one-quark and two-quark exchange diagrams that have to be evaluated for each two-body potential. The horizontal bars indicate the confinement, the one-gluon, one-pion, or one-sigma exchange interactions in eq.( 1).

where $P_{i j}^{O S T C}$ is the permutation operator of the $\mathrm{i}$-th and $\mathrm{j}$-th quark in orbital $(\mathrm{O})$ spinisospin (ST) and color space (C), respectively. The direct, as well as the one- and two-quark exchange contributions for the two-body potential of eq.(1) are depicted in fig.2. The solution for the unknown relative wave function $\chi_{L}(\mathbf{R})$ and the unknown eigenenergy is obtained from the variational principle

$$
\delta\left[\frac{\left\langle\Psi_{d^{\prime}}|H-E| \Psi_{d^{\prime}}\right\rangle}{\left\langle\Psi_{d^{\prime}} \mid \Psi_{d^{\prime}}\right\rangle}\right]=0,
$$

where the variation is with respect to the relative wave function $\chi_{L}(\mathbf{R})$. The results for the energy (mass) of the $d^{\prime}$ as well as for the harmonic oscillator parameter $b_{6}$ which minimizes the $d^{\prime}$ mass are shown in table 2 for the parameter sets of table 1 .

\subsection{Shell-Model Calculation for a $J^{P}=0^{-}, T=0$ six-quark system}

Next, we calculate the mass of the $d^{\prime}$-dibaryon in the translationally invariant shell-model (TISM) 14 15. Due to the negative parity of the $d^{\prime}$, only an odd number of oscillator quanta $N=1,3,5, \ldots$ is allowed. There is only one $N=1$ state which is compatible with $J^{p}=0^{-}, T=0$

$$
\left|\Psi_{d_{g . s .}^{\prime}}>=\right| N=1,[51]_{O},(\lambda \mu)=(10), L=1, S=1, T=0,[321]_{S T}>.
$$

For an unambigious classification of TISM states one has to specify the number of internal excitation quanta $N$, the Elliot symbol $(\lambda \mu)$, the Young pattern $[f]_{O}$ of the spatial permutational $S_{6}$-symmetry, further the total orbital angular momentum $L$, total spin $S$ and total isospin $T$ of the system. The specification of the intermediate $S U(4)_{S T}$ symmetry is necessary because in general, the same symmetry in $S T C$ space can be obtained from several states with different intermediate $S T$ symmetries. The mass of the $d^{\prime}$ is then given in first 
order perturbation theory by the expectation value of the Hamiltonian between the lowest harmonic oscillator state of eq.(11)

$$
M_{d^{\prime}}\left(b_{6}\right)=<\Psi_{d_{g . s .}^{\prime}}|H| \Psi_{d_{g . s .}^{\prime}}>
$$

In order to estimate the effect of configuration mixing with excited shell model states we include in addition ten $N=3$ states with orbital $[42]_{O}$ symmetry 15 . In this case also the $[51]_{S T},[411]_{S T},[33]_{S T},[321]_{S T}$, and $[2211]_{S T} S_{6}$ permutational symmetries are allowed.

With fixed parameters of the quark-quark interaction determined from eq.(7) we minimize the $d^{\prime}$ mass with respect to the harmonic oscillator parameter $b_{6}$ in the six-quark wave function. Note, that the harmonic oscillator parameter of the single baryon $(b)$ and the $d^{\prime}\left(b_{6}\right)$ wave function are different. The value of $b_{6}$ which minimizes the $d^{\prime}$ mass is a measure of the size of the system and is also given in table 3 .

Table 3: The mass $\left(M_{d^{\prime}}\right)$ and size $\left(b_{6}\right)$ of the $d^{\prime}$ in the six-quark shell model without and with configuration mixing.

\begin{tabular}{|c||c|c||c|c|}
\hline \multicolumn{1}{|c||}{} & \multicolumn{2}{c||}{$N=1$} & \multicolumn{2}{c|}{$N=1 \& N=3$} \\
\hline & $M_{d^{\prime}}^{(N=1)}[\mathrm{MeV}]$ & $b_{6}[\mathrm{fm}]$ & $M_{d^{\prime}}[\mathrm{MeV}]$ & $b_{6}[\mathrm{fm}]$ \\
\hline Set I & 2484 & 0.78 & 2413 & 0.78 \\
Set II & 2636 & 0.72 & 2553 & 0.73 \\
Set III & 2112 & 0.95 & 2063 & 0.96 \\
\hline
\end{tabular}

\section{Discussion and Summary}

As is evident from tables 2 and 3 the $d^{\prime}$ mass of the cluster model is lower than the single $N=1$ shell-model mass of eq.(12) but higher than the shell-model result with configuration mixing. Note that the $d^{\prime}$ mass calculated with set II (without pion and sigma-exchange between quarks) is some 150-200 MeV higher than the result with chiral interactions (set I). In any case, the calculated mass is about $350 \mathrm{MeV}$ higher than the value required by experiment. However, the confinement strength $a_{c}$ in the three-quark and six-quark system need not be the same. If we assume (set III) that $a_{c}$ in the six-quark system is weaker than in the nucleon one obtains considerably smaller values for $M_{d^{\prime}}$. This assumption is supported by the harmonic oscillator relation for $a_{c}$

$$
a_{c} \propto \frac{1}{m_{q} b^{4}} \frac{1}{N}
$$

which is inversely proportional to the number of quarks $N$ in the system. A weaker confinement strength is also expected due to the larger hadronic size of the $d^{\prime}\left(b_{6}\right)$ as compared to the hadronic size of the nucleon $(b)$. Set III differs from Set I of table 1 only in the strength of the parameter $a_{c}$ for which we take the value $a_{c}=5.0 \mathrm{MeV} / \mathrm{fm}^{2}$ in the six-quark calculation. Finally, both calculations give similar results for the $d^{\prime}$ mass and for its size. Let us briefly discuss the reasons for this. The outer product of the orbital [4] (tetraquark) and $[2]_{O}$ (diquark) symmetries gives the following six-quark symmetries

$$
[4]_{O} \otimes[2]_{O}=[42]_{O} \oplus \quad[51]_{O} \oplus[6]_{O}
$$


With the exception of the $[6]_{O}$ symmetry which is incompatible with $d^{\prime}$ quantum numbers these are also included in the enlarged $N=3$ shell-model basis 15 . Analogously, the outer product of the two clusters in spin-isospin space leads to

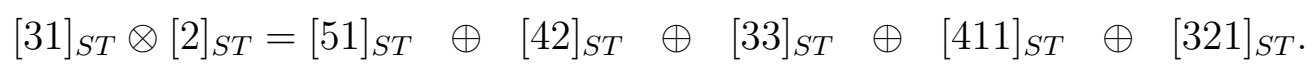

Comparison with eq.(10) in ref.14 shows that the $q^{4}-q^{2}$ cluster model wave function comprises the same $S_{6}$-symmetries in orbital and spin-isospin space (with the exception of the $[2211]_{S T}$ symmetry) as our enlarged shell model basis. Thus the trial function space spanned by both sets of basis functions is not very different.

In summary, we have calculated the mass of a $J^{P}=0^{-} T=0$ six-quark system in the NRQM using two different assumptions for the spatial distribution of the six quarks. The parameters have been determined from the constraints of eq.(7). As in our previous works 14, 15 our results are typically 300-400 MeV above the required resonance energy. However, for a weaker confinement strength $a_{c}$ in the six-quark system as suggested by eq.(13) we find a mass for the $d^{\prime}$ that is considerably smaller. The assumption of a weaker confinement strength in the six-quark system does not affect previous results of the model in the $B=2$ sector such as $N N$ scattering phase shifts or deuteron electromagnetic form factors which are completely insensitive to the model and strength of confinement 16.

\section{References}

1. R. Bilger, H. A. Clement and M. G. Schepkin, Phys. Rev. Lett. 71 (1993) 42; R. Bilger et al. Z. Phys. A343 (1992) 491

2. G. A. Miller, Phys. Rev. Lett. 53 (1984) 2008; M. Bleszynski and R. J. Glauber, Phys.Rev. C36 (1987) 681

3. M. A. Kagarlis and M. B. Johnson, Phys. Rev. Lett. 73 (1994) 38; N. Auerbach, W. R. Gibbs, J. N. Ginnochio, W. B. Kaufmann, Phys. Rev. C38 (1988) 1277

4. H. Garcilazo and L. Mathelitsch, Phys. Rev. Lett. 72 (1994) 2971

5. R. Bilger, H. A. Clement and M. G. Schepkin, Phys. Rev. Lett. 72 (1994) 2972

6. P. J. G. Mulders, A. Th. M. Aerts, and J. J. de Swart, Phys. Rev. D21 (1980) 2653; L. A. Kondratyuk, B. V. Martemyanov and M. G. Schepkin, Sov. J. Nucl. Phys. 45 (1987) 776

7. A. De Rujula, Howard Georgi, and S. L. Glashow, Phys. Rev. D12 (1975) 147

8. A. Manohar and H. Georgi, Nucl. Phys. B234 (1984) 189

9. A. M. Kusainov, V. G. Neudatchin, I. T. Obukhovsky, Phys. Rev. C44 (1991) 2343

10. F. Fernandez, A. Valcarce, U.Straub, and A. Faessler, J. Phys. G19 (1993) 2013; A. Valcarce, A. Buchmann, F. Fernandez, A. Faessler, Phys. Rev. C50 (1994) 2248

11. J. R. Bergervoet et al., Phys. Rev. C41 (1990) 143

12. A. Buchmann, E. Hernández and K. Yazaki, Phys. Lett. B269 (1991) 35; Nucl. Phys. A569 (1994) 661

13. Y. Yamauchi, A. Buchmann, Amand Faessler and Akito Arima, Nucl. Phys. A526 (1991) 495

14. L. Ya. Glozman, A. Buchmann and A. Faessler, J. Phys. G20 (1994) L49

15. Georg Wagner, L. Ya. Glozman, A. J. Buchmann and Amand Faessler, Nucl. Phys. A (1995) in press

16. K. Shimizu, Rep. Prog. Phys. 52 (1989) 1 